\title{
Approche linguistico-littéraire de: VICTOR HUGO: LES PAUVRES GENS, vers 1 à 43. \\ (La Légende des Siecles, 1859, 1877 et 1883)
}

\author{
Francisco RAMÓN TRIVES \\ Florentino HERAS \\ Universidad de Alicante
}

\section{TEXTE:}

1 Il est nuit. La cabane est pauvre, mais bien close'.

2 Le logis est plein d'ombre, et l'on sent quelque chose

3 Qui rayonne à travers ce crépuscule obscur².

4 Des filets de pêcheur sont accrochés au mur.

5 Au fond, dans l'encoignure où quelque humble vaisselle

6 Aux planches d'un bahut ${ }^{3}$ vaguement étincelle,

7 On distingue un grand lit aux longs rideaux tombants.

8 Tout près, un matelas s'étend sur de vieux bancs ${ }^{4}$,

9 Et cinq petits enfants, nid d'âmes ${ }^{5}$, y sommeillent.

10 La haute cheminée où quelques flammes veillent

11 Rougit le plafond sombre, et, le front sur le lit,

${ }^{1}$ Le détail est important; bien que pauvre, le pêcheur assure à sa famille un logis décent.

2 "Obscur": on n'allume la chandelle que lorsque c'est absolument nécessaire: le crépuscule obscur, signe de pauvreté, est combattu par ce "quelque chose" qui rayonne et évoque la lueur du foyer et la chaleur de l'affection.

${ }^{3}$ Ce buffet doit être un vaisselier.

${ }^{4}$ Et non sur un sommier; c'est l'une des conséquences de la pauvreté.

s Le matelas sert de "nid" à ces cinq âmes d'enfants qui s'éveillent à la vie. 
12 Une femme à genoux prie, et songe, et pâlit ${ }^{6}$.

13 C'est la mère. Elle est seule?. Et dehors, blanc d'écume,

14 Au ciel, aux vents, aux rocs, à la nuit, à la brume,

15 Le sinistre océan jette son noir sanglot.

16 L'homme est en mer. Depuis l'enfance matelot,

17 Il livre au hasard sombre une rude bataille.

18 Pluie ou bourrasque, il faut qu'il sorte, il faut qu'il aille,

19 Car les petits enfants ont faim. Il part le soir

20 Quand l'eau profonde monte aux marches du musoir ${ }^{8}$.

21 Il gouverne à lui seul sa barque à quatre voiles.

22 La femme est au logis, cousant les vieilles toiles,

23 Remmaillant les filets, préparant l'hameçon,

24 Surveillant l'âtre où bout la soupe de poisson,

25 Puis priant Dieu sitôt que les cinq enfants dorment.

26 Lui, seul, battu des flots qui toujours se reforment,

27 Il s'en va dans l'abîme et s'en va dans la nuit.

28 Dur labeur! tout est noir, tout est froid; rien ne luit.

29 Dans les brisants ${ }^{9}$, parmi les lames en démence,

30 L'endroit bon à la pêche, et, sur la mer immense,

31 Le lieu mobile, obscur, capricieux, changeant,

32 Où se plaît le poisson aux nageoires d'argent,

33 Ce n'est qu'un point; c'est grand deux fois comme la chambre ${ }^{10}$.

34 Or, la nuit, dans l'ondée et la brume, en décembre,

35 Pour rencontrer ce point sur le désert mouvant,

36 Comme il faut calculer la marée et le vent!

37 Comme il faut combiner sûrement les manoeuvres!

38 Les flots le long du bord glissent, vertes couleuvres;

39 Le gouffre roule et tord ses plis démesurés ble.

"Pâlit": en pensant à son mari qui est en mer, malgré la tempête épouvanta-

${ }^{7}$ Seule, parce que les enfants ne peuvent partager ses craintes.

${ }^{8}$ Extrémité de la jetée.

${ }^{9}$ Les récifs ou les écueils sur lesquels la mer vient se briser.

${ }^{10}$ Le pêcheur à l'âme simple emprunte ses comparaisons à ce qui lui est le plus familier. 
40 Et fait râler d'horreur les agrès effarés ${ }^{11}$.

41 Lui songe à sa Jeannie, au sein des mers glacées,

42 Et Jeannie, en pleurant, l'appelle; et leurs pensées

43 Se croisent dans la nuit, divins oiseaux du coeur.

En suivant l'histoire de l'Homme à travers les siecles, Victor Hugo est arrivé -dans La Légende des Siècles- à l'époque contemporaine, où il reconnaît une grandeur différente dans ses manifestations, mais non pas moins "sublime". On trouvera dans "Les pauvres gens" -objet de notre étude linguistico-littéraire- un aspect poétique de ce "sublime", où la forme et la musique pourront contribuer à la défense de cette classe si défavorisée.

Nous aborderons cette sorte d'épopée des "Pauvres Gens", en la situant d'abord dans un contexte historique, pour continuer notre étude suivant le plan déterminé par les quarante-trois vers que nous avons choisis. Nous ébaucherons une étude de l'article, de la qualification, du verbe, du participe présent, de la conjonction "et", de la versification, du timbre, des images et de la lumiere. Pour finir, nous indiquerons la bibliographie utilisée.

\section{LA FRANCE DU XIX ${ }^{\mathrm{e}}$ SIÈCLE.}

L'histoire de la France au XIXe siècle est d'une extrême complexité, et présente des contrastes très accusés: après l'épopée impériale, la Restauration; par deux fois, après la République, l'Empire, au début du siècle comme après 1848 .

A l'extérieur, des campagnes victorieuses, puis d'amères défaites, et deux invasions (1814-1815 et 1870).

A l'intérieur, des bouleversements politiques fréquents, des révolutions, de nombreux mouvements sociaux. Tant d'événements ont exercé une influence déterminante sur l'évolution littéraire du siècle,

11 Même les objets inanimés paraissent vivre dans l'horreur de la tempête. Les voiles et les cordages du navire semblent épouvantés par la profondeur des vagues et la violence du vent. 
dont les lignes de force n'ont plus la relative simplicité de celles des siècles précédents, plus aisées à définir.

Le XIXe siècle revendique hautement le libéralisme en littérature: liberté d'inspiration et de forme - donc recherche de thèmes nouveaux et d'expressions originales - liberté de s'exprimer soi-même, d'aborder de front les problèmes politiques et sociaux, d'associer la science à l'oeuvre d'art et même à la póésie.

Les intentions sont d'une largeur et d'une hardiesse extrêmes; le désir de renouvellement anime tout le siècle, et la diversité des tendances comme des oeuvres est caractéristique d'une continuelle transformation.

L'époque offre, en effet, des conditions sociales toutes nouvelles dont les écrivains doivent tenir compte: la Révolution a transformé la société, et les lecteurs sont devenus plus nombreux. Ce n'est plus à une élite que l'auteur s'adresse, mais à un large public; d'où le succès du théâtre et du roman. Le développement industriel et la concentration urbaine qu'il provoque posent des questions sociales de plus en plus aiguës; l'enrichissement de la bourgeoisie donne une place essentielle au problème de l'argent, qu'abordent surtout les romanciers. La misère de certaines classes sociales (comme celle des pêcheurs, dans le poème qui nous occupe) devient à son tour un thème littéraire, et le libéralisme politique s'affirme dans de très nombreuses oeuvres, même poétiques.

Des sentiments nouveaux -ou renouvelés- apparaissent sous les formes les plus diverses: le sentiment de la nature, étendu à des décors jusque-là dédaignés ou inconnus (la mer, les forêts sombres, les paysages lointains) s'élargit jusqu'à un exotisme parfois déroutant. Le sentiment religieux est réintégré par les romantiques dans l'oeuvre littéraire. La curiosité historique et scientifique oriente l'inspiration vers les civilisations ignorées ou méconnues, offrant des éléments originaux et authentiques de couleur locale. L'étude personnelle s'enrichit de nuances individuelles parfois étranges et rares. Enfin, le sens social s'affirme d'autant plus nettement que les auteurs participent souvent aux luttes politiques. Député, Lamartine devient, en 1848, le chef du Gouvernement Provisoire. Victor Hugo s'exile après le coup d'Etat du 
2 décembre 1851. Plus tard, Zola défend courageusement l'innocence de Dreyfus ${ }^{12}$.

Aussi la littérature du XIXe siècle est-elle très riche et très diverse. Toutes les formes s'y rencontrent: poésie, thêtre, roman, histoire, critique... Souvent, un même auteur s'affirme dans plusieurs genres à la fois.

L'évolution des idées et des goûts permet de distinguer trois grands courants qui paraissent se succéder, les deux derniers se déterminant par réaction contre les tendances précédentes, sans que leurs dates puissent être fixées de manière absolue. De 1820 à 1850 , le Romantisme, dont l'influence a marqué tout le siècle. De 1850 à 1870 environ, le Réalisme, qui renouvelle surtout le thêâtre et le roman, mais qui touche aussi la poésie parnassienne. Après 1870, le Symbolisme, qui s'efforce d'exprimer des rêves, des nuances, et de rendre à l'art sa subtilité et son mystère. Ses deux domaines d'élection sont la poésie et la musique.

Le mouvement romantique a eu une ampleur et une puissance extraordinaires. Né avec Chateaubriand, il éclate triomphalement d'abord avec les Méditations de Lamartine, en 1820, puis surtout avec les oeuvres de Victor Hugo, de Vigny et de Musset. Si la poésie est son mode d'expression préféré, il s'affirme aussi par le thêâtre avec le drame, et par le roman. Victor Hugo apparaît très tôt comme le "chef de file" du mouvement, parce qu'il exprime hardiment les ambitions et les principes, et qu'il a laissé l'oeuvre la plus abondante du siecle. Le Romantisme proclame les droits de l'imagination et du coeur et exalte le "moi" tourmenté et orgueilleux d'êtres sensibles qui ont souvent le goût du malheur.

Toute oeuvre romantique est donc lyrique, le drame comme le roman. L'auteur veut s'y exprimer librement, sans plus se lier à des genres et à des règles déterminés; mais il reste toujours un artiste soucieux de belles images, de rythmes nouveaux, de rimes sonores.

De grands peintres, aux compositions puissantes et dramatiques, aux coloris éclatants, achèvent d'illustrer cette période: ainsi Géricault et Delacroix.

12 Dreyfus F.G. (1968): Histoire Universelle: "Le temps des révolutions, 1787-1870". Livre de Poche/Larousse. 
Cette vue d'ensemble ne suffit pas, en réalite, à rendre compte de la diversite d'un siecle qui voit s'opposer les théories de la "fonction du poète" (chargé d'éclairer les foules) et de "l'art pour l'art" (l'oeuvre ne doit tendre qu'à là beauté), qui voit apparaître tour à tour la poésie lyrique, épique et satirique, les romans sociaux, les drames et les pièces à thèse; qui représente côte à côte l'oeuvre démesurée, formant un ensemble ambitieux (Balzac, Zola) et le recueil étroit qui se veut parfait (Les Trophées de J.M. de Hérédia).

Notre époque doit au XIXe siècle des sources d'inspiration, des thèmes et des moyens d'expression dont l'influence est encore sensible dans le domaine littéraire, musical et pictural. Il lui doit une certaine conception de l'homme et de l'art, qui est aussi la nôtre. Hugo devient ainsi notre contemporain et ses "pauvres gens", nos proches.

\section{L'ARTICLE}

L'article, qui existait en grec ancien, était inconnu au latin classique. C'est une creation du bas-latin, qui, après quelques hésitations entre plusieurs particules, a tiré l'article défini du démonstratif affaibli "ille" (renforcé, comme démonstratif, par l'adjonction d'une particule: "ecce-ille"): "le", "la", "les" sont les formes atones de (il)lum, (il)la, (il)los. De façon parallèle, l'indéfini a dégagé sa fonction du numéral "un", qui a conservé conjointement sa valeur primitive.

Quant au partitif, il s'est formé par étapes successives qui attestent les tendances du français vers une précision accrue. Cette fonction s'exprimait en latin sans particule, par simple adjonction du nom complément au verbe: "bibere aquam", en très ancien français: "boire eau" (l'italien en est resté à cette étape: bere acqua). Puis, la préposition s'introduit, pour marquer qu'il ne s'agit que d'une fraction, et non de la totalité de la substance ou de l'objet: "boire d'eau", deuxième étape à laquelle s'est arrêtée la langue d'oc ("beure d'aigo"). Enfin, insertion de l'article défini, pour mieux préciser l'objet: "boire de l'eau"13.

13 Dauzat Albert (1947): Grammaire raisonnée de la Langue Française. Paris: Edition IAC. 


\subsection{Valeur morphologique.}

L'article marque le genre et le nombre. Il permet le changement de catégorie grammaticale. Il traduit particulièrement bien l'infini, l'inconnu, l'invraisemblable, ce qui permet de donner au discours des résonnances philosophiques.

\subsection{Emploi des différents articles.}

L'article actualise le substantif. Soit sous sa forme entiere: "la cabane" (v.1); soit sous forme élidée: "l'encoignure" (v.5); soit sous forme contractée: "du bord" (v.38).

L'article sert aussi à introduire une notion de réalité immédiatement tangible, ou perçue concrètement: "la nuit", toujours la nuit. Le singulier "l'homme est en mer"(v.16) signale que le concept est pris dans sa totalité par rapport à sa compréhension et non à son extension.

1.3. L'article indéfini actualise un ou des objets pris dans un ensemble d'objets du même ordre.

Il sert à présenter un objet non encore connu: "une rude bataille" (v.17). Il sert aussi à actualiser avec une totale indifférence: "au sein des mers glacées" (v.41). Victor Hugo veut respecter cette idée de mystère qu'a la mer en soi, en nous donnant un sème de l'infini, qui irait de pair avec l'invraisemblable conceptuel inhérent de ce vers (v.41).

\subsection{Article zéro.}

Devant un substantif pris en fonction d'adjectif: "matelot" (v.16).

\subsection{Les autres déterminants.}

\subsubsection{Les démonstratifs.}

"Qui rayonne à travers ce crépuscule obscur" (v.3). De prime abord, on dirait que ce démonstratif a une valeur d'article défini, qui correspondrait à "le". Mais une lecture plus attentive permet d'affirmer que Victor Hugo veut mettre en relief "le crépuscule", en l'approchant de lui: d'où l'emploi de "ce", qui serait finalement plus vraisemblable (l'adjectif déterminatif démonstratif serait très proche du "je", donc de l'auteur). 
1.5.2. Le déictique neutre.

"ce n'est qu'un point"(v.33). La neutralité nous donne un appui vers l'invraisemblable ou le monde imaginaire que defend Victor Hugo. Nous pouvons déduire que l'abondance d'articles, dans leur diversité, contraste bien avec le déictique démonstratif: "ce crépuscule obscur" souligne cette idée chère aux romantiques de la présence du "cosme" se mêlant à la présence de l'auteur - dans toute oeuvre littéraire.

\section{LA QUALIFICATION}

L'actualisation par l'article est affaire d'instinct et d'automatisme. Mais on peut aussi noter les qualités essentielles ou accessoires de la chose ou du concept: la qualification dépend du sujet parlant. Victor Hugo en use largement dans ce texte.

2.1. Nature des agents de qualification.

\subsubsection{L'adjectif:}

"pauvre" (v.1); "seule" (v.13); "sombre" (v.17); "noir" (v.28); "froid" (v.28).

\subsubsection{Epithete:}

"un grand lit aux longs rideaux tombants" (v.7); "sur de vieux bancs" (v.8); "la haute cheminée" (v.10); "blanc d'écume" (v.13); "le sinistre océan jette son noir sanglot" (v.15); "une rude bataille" (v.17); "car les petits enfants ont faim" (v.19); "dur labeur!" (v.28); "vertes couleuvres" (v.38); "divins oiseaux du coeur" (v.43).

\subsubsection{Participes présents, adjectifs verbaux:}

"aux longs rideaux tombants" (v.7); "le lieu mobile, obscur, capricieux, changeant" (v.31); "sur le désert mouvant" (v.35).

2.1.4. Participe passé passif:

"plis démesurés" (v. 39); "les agrès effarés" (v.40).

2.1.5. Substantif + substantif: 
"l'enfance matelot" (v.16). C'est une image semblable à: "pâtre promontoire", "astres-hiboux" (Voir ailleurs dans La Légende des Siecles).

\subsubsection{Substantif précédé d'une préposition:}

"blanc d'écume" (v.14): hébraïsme du type "Dieu de gloire", usuel depuis le Moyen-Age. Le tour hébraïque s'est répandu en France grâce à sa ressemblance -toute superficielle du reste- avec d'autres constructions héritées ou non du latin: "Vir magni ingenii". On doit le considérer surtout comme un artifice de style destiné à introduire dans la phrase un élément de variété, en évitant l'accumulation des épithètes ou des symétries trop accusées; un moyen commode, aussi, de reproduire le mouvement constant du flux et du reflux de l'océan, en se servant de cette valeur chromatique du "blanc", pour réveiller notre sensibilité a partir de notre vue.

\subsubsection{La proposition relative:}

"Et l'on sent quelque chose / qui rayoune à travers ce crépuscule obscur" (v.2-3). Au lieu d'une simple touche nous avons affaire à un élargissement de la vision. La qualification s'anime grâce au verbe et au tableau qui l'accompagne.

\subsection{Valeur stylistique des agents de qualification.}

Les adjectifs, ici, sont non pas des adjectifs de détermination (ex: "un stylo noir", par rapport à "un stylo bleu"), mais des adjectifs de caractérisation, servant à noter certains caractères de l'objet ou du concept qu'il représente: "ce crépuscule obscur" montrant ainsi le caractère dramatique de ce poème.

\subsection{Valeur sémantique.}

L'épithète est à la fois, chez Victor Hugo, l'expression d'une vision, d'une audition et d'un jugement; une impression intellectuelle se superpose à une image soit visuelle soit auditive: "et dehors, blanc d'écume $/ / \ldots$ le sinistre océan jette son noir sanglot" (v.15). 
2.4. Mise en relief de la qualification.

Le nombre et la variété des modes de qualification attirent déja l'attention. La longueur de certains termes leur donne aussi de l'importance: "capricieux" (v.31). De même, l'usage au figuré de certains adjectifs: "le désert mouvant" (v.35).

\subsection{La place.}

Le plus souvent, en français, l'épithète physique à valeur descriptive se trouve postposée. Notons, cependant: "quelque humble vaisselle"

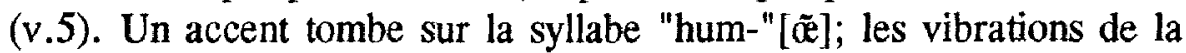
nasale mais aussi l'allongement et le chuchotement soulignent bien l'idée.

"Ses agrès effarés"(v.40): la versification interdit "ses effarés agrès"; mais, en outre, on remarque le souci de varier la symétrie dans le groupe nom + adjectif, suivi de: adjectif + nom. Le procédé est usuel depuis Chateaubriand.

\subsection{Les inversions,}

"une rude bataille" (v.17); "battu des flots" (v.26). Cette licence poétique, très consciente chez Hugo, qui s'est expliqué là-dessus (dans une lettre à W. Tenint) met l'accent autant sur l'adjectif que sur le complément.

La place des adjectifs, enfin: Hugo leur accorde souvent des places privilégiées.

Soit en tête du vers ("Dur labeur!", v.28).

Soit à la rime ("ce crépuscule obscur", v.3).

Soit à la césure ("rougit le plafond sombre", v.11).

En guise de conclusion, on pourrait déduire, d'après l'étude de ce poème, que dans une structure fermée -la poésie-, pour des raisons de musicalité, la place de l'adjectif qualificatif est imposée par le message intentionnel de l'auteur. Or, nous serions d'accord avec la thèse de Spang-Hanssen $(1967,61)$ quand il nous fait part de la complexité des règles qui fixent la place de l'adjectif dans ses diverses fonctions. En effet, Hanssen affirme: 
"Etant donné la complexité des règles qui fixent la place de l'adjectif épithète, on ne peut pas s'étonner que les grammairiens du français moderne négligent un peu le problème mineur de la combinaison de deux ou plusieurs adjectifs auprès du nom. Si les deux adjectifs se répartissent harmonieusement, un de chaque côté du substantif, il n'y a aucun problème nouveau; mais si, au contraire, les deux adjectifs, selon les règles générales, devraient se placer du même côté, les difficultés surgissent. Les indications que fournissent les grammaires sont des plus sommaires en ce qui concerne la postposition, trop sommaires, surtout, si l'on prend en considération l'importance du problème" 14 .

\section{LE VERBE}

Nous abordons l'étude du verbe selon les criteres d'Albert Dauzat, dans sa Grammaire raisonnée de la langue française.

Dauzat affirme:

"le verbe exprime une action ou un état en rapport avec l'être ou l'objet qui le conditionne ou qui le subit. Cette relation est omise dans la plupart des grammaires: on oublie volontiers que le verbe considéré en soi est une abstraction de grammairien et que, dans le langage, il apparaît toujours en fonction d'un sujet. Le besoin de sujet est réduit au minimum et tend vers zéro avec le sujet purement grammatical des verbes impersonnels: il pleut, il faut (populairement et familièrement: faut). Seul le substantif exprime une action ou un état en soi (l'action, l'état).

Et il ajoute:

"Grammaticalement, le verbe forme, en principe, le centre de la phrase: si le substantif (ou pronom) sujet est le chef qui la commande (ce que confirme l'accord), le verbe est le moteur qui transmet les ordres, qui ordonne la structure, en reliant le sujet aux attributs ou aux compléments. - Il arrive d'ailleurs fréquemment que le verbe commence la

${ }^{14}$ Cité par Lago, J. (1986): La acumulación de adjetivos calificativos en la frase nominal del francés contemporáneo. Universidade de Santiago de Compostela. 
phrase (avec l'impératif qui n'a pas de sujet exprimé: "laissez venir à moi les petits enfants", ou dans des formules expressives: "Restait cette redoutable infanterie de l'armée d'Espagne" (Bossuet), ou qu'il la termine, quand le verbe ne comporte pas de complément ou que les compléments le précèdent ("notre ami est arrivé"; "hier, à trois heures, notre ami est arrivé").

Appliquant ces critères à l'étude des 43 vers que nous avons choisis, nous avons fait le classement suivant: transitifs, intransitifs, voix moyenne, verbes de mouvement, et les effets de style demandés pour des raisons poétiques.

\subsection{Transitifs.}

Dans cette étude, nous avons souligné le sémantisme imposé par le système contextuel dont l'importance essentielle se trouverait dans l'aspect de nos sens -vue, ouie- comme les deux véhicules principaux pour avoir accès à notre âme sensible. En plus, en utilisant des verbes polysémiques, Hugo déclenche, chez le lecteur, cette ambiguïté interprétative.

Vers 2: "et l'on sent quelque chose". Le verbe "sentir" pourrait vouloir dire "apercevoir, regarder et, à la rigueur, sentir un parfum". En un mot, nous serions en présence d'un substitut du verbe "voir". De plus, le fait d'employer le pronom "on" impersonnel, mais à la fois très polysémique ( $=$ je, vous, nous, eux, etc.), établit un parallelisme logique avec le verbe "sentir", dont la ou les valeur(s) ont été décrites ci-dessus.

Vers 7: "On distingue un grand lit". On dirait que le verbe "distinguer" serait plus proche de l'auteur que le verbe "sentir", mais tous les deux sont précédés d'un impersonnel à valeur polysémique. En même temps, il y a comme une sorte de voile où se cache le DieuCréateur, "l'Auteur".

Vers 10-11: "La haute cheminée rougit le plafond sombre". Bien que, dans la langue générale, le verbe "rougir" ait une valeur chromatique, dans ce contexte précis le verbe aurait un équivalent du type lumineux: "éclairer à peine, de façon atténuée", comme antithèse de l'épithète "sombre". 
Vers 12: "Une femme à genoux prie". Ce verbe peut être employé soit avec complément d'objet direct exprimé (Dieu), soit avec un C.O.D. non-exprimé. C'est le cas choisi ici par le poète.

Vers 15: "Le sinistre océan jette son noir sanglot". Par un artifice poétique l'auteur détruit la structure normale du verbe, en commençant par cette sorte de "datif" ou bénéficiaire ${ }^{15}$, provoquant un effet de style et de mise en relief des éléments cosmiques: "Au ciel, aux vents, aux rocs, à la nuit, à la brume" (v.14).

Vers 17: "Il livre au hasard sombre une rude bataille". Nous avons un verbe d'une caractéristique semblable à celui du vers 15 , en y ajoutant cette idée presque obsessive du manque de lumière: "sombre" marquant ou annonçant ainsi le lieu physique où "cette rude bataille" va avoir lieu, c'est-à-dire, la mer du Nord, et le climat plutôt grisâtre de cette mer qui baigne l'île où Victor Hugo est exilé. Cette réminiscence personnelle aurait pu avoir une certaine influence dans l'aspect descriptif avec quelques petites touches émotives hugoliennes. Ce qui frappe dans ce vers, c'est l'emploi emphatique "au hasard sombre", teinté de ces sèmes imposés par le destin incertain, ingrat, tragique ou parfois providentiel, propre à la vie des pêcheurs.

\subsection{Intransitifs.}

Nous nous limiterons à signaler quelques-uns des exemples les plus significatifs.

Vers 3: "Qui rayonne à travers ce crépuscule obscur". Le verbe "rayonner" nous donne une idée de lumière plutôt forte, mais Hugo se charge de faire atténuer cette lumière à l'aide de ce syntagme prépositionnel avec l'antithèse "obscur" précédé de l'acte cosmique de décrépitude ou coucher de soleil, réussissant ainsi cette idée obsessive de tout le poeme: l'absence de lumière ou la valeur sombre sur l'aspect chromatique et conceptuel du message tragique de la destinée de pêcheur.

Vers 10: "quelques flammes veillent". Le verbe "veiller" suppose un être animé, plus ou moins humain, alors que nous assistons à une personnification de "quelques flammes", qui ont une double valeur,

${ }^{15}$ Se référer à l'étude des cas réalisée par B. Pottier. 
physique et de chaleur humaine, d'où l'importance de ce symbolisme qui dénote toujours cette lumière - sombre, malgré tout, et constante.

3.3. L'intentionnalité des verbes de mouvement.

Le mouvement chez Hugo est toujours présent, à l'aide de verbes dont le sémantisme est inhérent.

Vers 18-19: "il faut qu'il sorte, il faut qu'il aille"...;

"Il part le soir..."

Vers 27: "Il s'en va dans l'abîme et s'en va dans la nuit".

Non seulement tous ces verbes nous donnent la vision de mouvement, mais aussi et surtout l'idée d'obligation paternelle pour gagner le pain pour ses enfants, malgre le caractère hasardeux du travail a accomplir. Cette idée dramatique est exprimée directement par "abîme", et symboliquement, par "nuit".

\subsection{Le passif.}

Vers 4: "Des filets de pêcheur sont accrochés au mur". C'est le seul passif de tout le poème, dont le complément agent est absent pour mieux marquer l'anonymat du fait accompli. En l'occurrence, on suppose que c'est quelqu'un d'animé et d'humain. On dirait que les filets sont là comme une sorte d'ornement et de carte d'identité du pêcheur.

3.5. L'emploi du verbe "être".

3.5.1. Attribut.

"la cabane est pauvre (V.1)

"elle est seule" (v,13)

"tout est noir, tout est froid" (v.28)

3.5.2. Copule.

Traduisant l'espace: "Le logis est plein d'ombre" (v.2)

"L'homme est en mer" (v.16)

"La femme est au logis" (v.22)

Traduisant le temps météorologique:

"Il est nuit" (v.1) 


\subsubsection{Présentatif.}

"c'est la mère" (v.13)

"c'est grand deux fois comme la chambre" (v.33)

"ce n'est qu'un point" (v.33)

3.5.4. Elliptique.

"...mais bien close" (v.1)

"depuis l'enfance matelot" (v.16)

3.5.5. Passif.

"des filets sont accrochés au mur" (v.4)

\subsection{La participe présent}

Albert Dauzat donne, du participe présent, la définition suivante:

"Le participe présent est invariable quand il conserve sa valeur verbale. Ainsi en a décidé l'Académie française en 1679 , d'après un usage qui commençait à s'établir, sous l'influence de l'emploi en gérondif (invariable) du participe présent; c'est une des rares décisions grammaticales de l'Académie. L'usage ancien, hérité du latin, qui faisait accorder en nombre le participe, est encore fréquent chez les classiques du XVIlle siècle: "Les morts se ranimants à la voix d'Elisée" (Racine: Athalie, I,1).

Il en est resté quelques vestiges dans la langue juridique (les ayants droit) ou dans des locutions clichées (toute affaire cessante, à la nuit tombante, séance tenante) ${ }^{116}$.

Cette distinction permet de séparer le participe à valeur verbale du participe adjectivé; ce n'est parfois qu'une nuance, mais que la pause ou l'absence de pause rend sensible: "nous avons vu cette femme tremblante de peur" ou: "nous l'avons vue, cette femme, tremblant de peur". L'orthographe distingue parfois les deux valeurs, quand l'adjectif est devenu plus ou moins indépendant du participe, notamment pour les finales - quant, -guant; les adjectifs: -cant, -gant. Comparer: "ce

${ }^{10}$ Dauzat, A.: Op. cit. 
raisonnement ne convainquant personne, on a passé à un autre sujet" et: "on a tenu un raisonnement peu convaincant".

Vers 22: ..." cousant les vieilles toiles".

Vers 23: "Remmaillant les filets, préparant l'hameçon"

Vers 24: "Surveillant l'âtre...

Vers 25: "Puis priant Dieu sitôt que les enfants..."

Victor Hugo se charge de nous décrire la femme du pêcheur avec ses occupations non seulement domestiques, mais aussi professionnelles, voire maternelles. L'emploi du participe présent nous donne cette vision dure et constante de mouvement progressif, personnel et symbolique; le poète montre ainsi la vie quotidienne d'une femme de pêcheur. Ce faisant, l'auteur nous émeut et nous donne une certaine conscience de la problématique de cette classe sociale si misérable, abandonnée par les classes dominantes. Or, le choix de tous ces verbes établit un lien logique entre les différentes tâches accomplies par Jeannie.

3.7. La conjonction "et".

\begin{tabular}{|c|c|c|c|}
\hline VERS & QTÉ & $\begin{array}{c}\text { ORDRE DANS } \\
\text { LA PHRASE }\end{array}$ & $\begin{array}{c}\text { VALEUR } \\
\text { SÉMANTIQUE }\end{array}$ \\
\hline $1-4$ & 1 & après une virgule & adversative $=$ mais \\
\hline $5-8$ & 0 & & \\
\hline $9-12$ & 4 & $\begin{array}{l}\text { début } \\
\text { virgule } \\
\text { incise }\end{array}$ & $\begin{array}{l}\text { emphatique (v.9) } \\
\text { emphatique (v. } 11) \\
\text { passe-partout et mise en } \\
\text { relief. } \\
\text { valeur formelle (requise } \\
\text { par la versification) }\end{array}$ \\
\hline $13-16$ & 1 & début de phrase & $\begin{array}{l}\text { mise en relief du syntag- } \\
\text { me nominal: "océan" }\end{array}$ \\
\hline $17-20$ & 0 & & \\
\hline
\end{tabular}




\begin{tabular}{|c|c|c|c|}
\hline VERS & QTÉ & $\begin{array}{l}\text { ORDRE DANS } \\
\text { LA PHRASE }\end{array}$ & $\begin{array}{l}\text { VALEUR } \\
\text { SÉMANTIQUE }\end{array}$ \\
\hline $21-24$ & 0 & & \\
\hline $25-28$ & 1 & $\begin{array}{l}\text { début deuxième } \\
\text { hémistiche }\end{array}$ & $\begin{array}{l}\text { valeur stylistique d'addi- } \\
\text { tion }\end{array}$ \\
\hline $29-32$ & 1 & incise, virgule & $\begin{array}{l}\text { mise en relief du syntag- } \\
\text { me nominal, et coupure de } \\
\text { l'alexandrin }\end{array}$ \\
\hline $33-36$ & 2 & $\begin{array}{l}\text { début } 2 \mathrm{e} \text { hémis- } \\
\text { tiche. accent sur } \\
\text { le pied }\end{array}$ & $\begin{array}{l}\text { hétérogénéité } \\
\text { addition = "en plus" }\end{array}$ \\
\hline $37-40$ & 2 & $\begin{array}{l}\text { début de phrase. } \\
\text { accent porté sur } \\
\text { le } 5 \text { e pied (v.39), } \\
\text { afin de ne pas } \\
\text { répéter le sujet }\end{array}$ & $\begin{array}{l}\text { addition avec un sème } \\
\text { supplémentaire ayant une } \\
\text { valeur stylistique }=\text { force }\end{array}$ \\
\hline $41-43$ & 2 & $\begin{array}{l}\text { début de phrase. } \\
\text { accent porté sur } \\
\text { le } 9 \text { e pied }(v .42)\end{array}$ & $\begin{array}{l}\text { mise emphatique et donc } \\
\text { mise en relief de Jeannie }\end{array}$ \\
\hline
\end{tabular}

De la quantité de conjonctions "et" utilisées par Victor Hugo, on pourrait déduire que le "relateur" "et" est, sans aucun doute, très musical, puisqu'il s'agit d'une voyelle orale simple dont le timbre est le plus aigu de la langue française après le " $\mathrm{i}$ ". Sa sonorité est donc très agréable à l'oreille. En plus, sa valeur sémantique est très polyvalente, d'après ce qu'on vient de constater dans ce poème. En effet, cette polyvalence se trouve présente dans le tableau ci-dessus: depuis sa valeur adversative jusqu'à une valeur "zéro" rhétorique ou parfois comme souffle poétique. Sans oublier sa valeur d'addition et d'autres sèmes qui renforceraient l'idée stylisée de la conjonction "et". 
Francisco RAMÓN TRIVES Y FloRENTINO HERAS

\section{VERSIFICATION}

\begin{tabular}{|c|c|c|c|}
\hline VERS & $\begin{array}{l}\text { ACCENTS } \\
\text { PROSODIQUES }\end{array}$ & $\begin{array}{l}\text { HEMIS- } \\
\text { TICHES }\end{array}$ & $\begin{array}{l}\text { TERMINAISON } \\
\text { / RIME }\end{array}$ \\
\hline 1 & $3,6,9,12$ & 6 & close \\
\hline 2 & $3,6,9,12$ & 6 & chose \\
\hline 3 & $3,6,10,12$ & 6 & obscur \\
\hline 4 & $3,6,10,12$ & 6 & mur \\
\hline 5 & $2,6,10,12$ & 6 & vaisselle \\
\hline 6 & $3,6,9,12$ & 6 & étincelle \\
\hline 7 & $3,6,10,12$ & 6 & tombants \\
\hline 8 & $2,6,8,12$ & 6 & bancs \\
\hline 9 & $1,6,9,12$ & 6 & sommeillent \\
\hline 10 & $3,6,10,12$ & 6 & veillent \\
\hline 11 & $2,6,7,12$ & 6 & lit \\
\hline 12 & $3,6,9,12$ & 6 & pâlit \\
\hline 13 & $3,6,9,12$ & 6 & ecume \\
\hline 14 & $2,4,6,9,12$ & 6 & brume \\
\hline 15 & $3,6,8,10,12$ & 6 & sanglot \\
\hline 16 & $4,9,12$ & 9 & matelot \\
\hline 17 & $2,6,9,12$ & 6 & bataille \\
\hline 18 & $1,4,8,12$ & 8 & il aille \\
\hline 19 & $1,5,7,12$ & 7 & le soir \\
\hline
\end{tabular}


APPROCHE LINGUISTICO-LITTÉRAIRE...

\begin{tabular}{||l|l|l|l||}
\hline VERS & $\begin{array}{c}\text { ACCENTS } \\
\text { PROSODIQUES }\end{array}$ & $\begin{array}{c}\text { HEMIS } \\
\text { TICHES }\end{array}$ & $\begin{array}{c}\text { TERMINAISON } \\
\text { / RIME }\end{array}$ \\
\hline 20 & $1,5,7,12$ & 7 & musoir \\
\hline 21 & $3,6,9,12$ & 6 & voiles \\
\hline 22 & $2,6,8,12$ & 6 & toiles \\
\hline 23 & $3,6,9,12$ & 6 & l'hameçon \\
\hline 24 & $3,4,5,6,9,12$ & 6 & poisson \\
\hline 25 & $1,4,6,9,12$ & 6 & dorment \\
\hline 26 & $1,2,4,6,9,12$ & 6 & se reforment \\
\hline 27 & $3,6,9,12$ & 6 & la nuit \\
\hline 28 & $3,6,9,12$ & 6 & luit \\
\hline 29 & $4,6,9,12$ & 6 & en démence \\
\hline 30 & $2,6,7,10,12$ & 6 & immense \\
\hline 31 & $2,5,7,10,12$ & 5 & changeant \\
\hline 32 & $3,6,10,12$ & 6 & d'argent \\
\hline 33 & $4,6,8,12$ & 6 & chambre \\
\hline 34 & $1,3,6,9,12$ & 6 & décembre \\
\hline 35 & $4,6,10,12$ & 6 & mouvant \\
\hline 36 & $1,6,9,12$ & 6 & vent \\
\hline 37 & $1,6,9,12$ & 6 & manoeuvres \\
\hline 38 & $2,4,6,7,12$ & 7 & couleuvres \\
\hline 39 & $3,6,8,12$ & 6 & démesurés \\
\hline 40 & $4,6,9,12$ & 6 & effarés \\
\hline
\end{tabular}




\begin{tabular}{||c|l|l|l||}
\hline VERS & $\begin{array}{c}\text { ACCENTS } \\
\text { PROSODIQUES }\end{array}$ & $\begin{array}{c}\text { HEMIS- } \\
\text { TICHES }\end{array}$ & $\begin{array}{c}\text { TERMINAISON } \\
\text { / RIME }\end{array}$ \\
\hline 41 & $1,6,8,12$ & 6 & glacés \\
\hline 42 & $3,6,8,12$ & 6 & pensées \\
\hline 43 & $3,6,10,12$ & 6 & coeur \\
\hline
\end{tabular}

4.1. Tous les vers ont, en principe, un hémistiche au 6ème vers, marquant ainsi la coupure; et la terminaison de la rime se trouve au

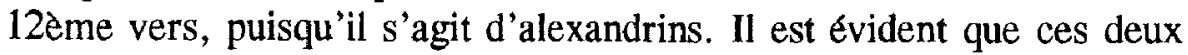
rimes contiennent les idées fondamentales.

4.2. Le compte des syllabes:

diérèse: "océan" (v.15)

synérèses habituelles: "nuit" (répétées plusieurs fois), "luit" (v.27).

4.3. Le rythme des alexandrins:

Tous ont un accent, si faible soit-il, à la 6ème syllabe (v.1: "cabane").

$\mathrm{La}$ valeur des monosyllabes renforce l'idée cosmique et le déterminisme du pêcheur face à sa destinée: "ciel, vents, rocs, nuit" (v.14-15)

D'autres vers reposent sur une structure classique:

..."Et dehors, blanc d'écume /.../

Le sinistre océan jette son noir sanglot" ( $\mathrm{v}-13$ et 15$)$.

\subsection{La rime}

En général, la rime utilisée par Victor Hugo est une rime suffisante, puisqu'elle est portée par deux sons.

On assiste aussi généralement à l'alternance des rimes masculines et féminines: $\mathrm{AA}, \mathrm{BB}$. 


\subsection{L'hémistiche}

Comme nous l'avons déjà indiqué, dans tous ces vers alexandrins, l'hémistiche est en général régulier et placé sur la 6ème syllabe, sauf dans les vers suivants:

\begin{tabular}{|c|l|l|}
\hline VERS & \multicolumn{1}{|c|}{ HEMISTICHE } & \multicolumn{1}{|c|}{ MOT } \\
\hline 16 & 9 & l'enfance \\
\hline 18 & 8 & sorte \\
\hline 19 & 7 & faim \\
\hline 20 & 7 & monte \\
\hline 31 & 5 & obscur \\
\hline 38 & 7 & glissent \\
\hline
\end{tabular}

Toutes ces césures sont imposées par la structure syntactique et la valeur sémantique. Néanmoins, dans le vers 38 , nous avons choisi la syllabe 7 car on est en présence d'une image très importante. En effet, dans l'assimilation "flots = couleuvres", la syllabe "glissent" est inhérente au sifflement provoqué par les fricatives alvéolaires-dentales sourdes, dont la sonorité assourdie ressemble ou imite le sifflement des couleuvres. Il y a donc une symbiose entre le signifiant et le signifié, marquant ainsi une image très auditive fondée sur l'onomatopée animaux-reptiles.

Nous aurions aimé pouvoir étudier la valeur musicale des allongements dans les terminaisons des 43 vers, mais nous sommes limités par l'espace disponible.

\section{LE TIMBRE}

\begin{tabular}{|c|l|l|l|l||}
\hline VERS & \multicolumn{3}{|c|}{ ACCENTS } \\
\hline & TRES AIGU & MOINS AIGU & $\begin{array}{c}\text { MOINS GRAVE } \\
\text { SOMBRE }\end{array}$ & PLUS GRAVE \\
\hline 1 & 3 & & 9,12 & 6 \\
\hline 2 & 3 & & 6,12 & 9 \\
\hline
\end{tabular}




\begin{tabular}{|c|c|c|c|c|}
\hline \multirow[t]{2}{*}{ VERS } & \multicolumn{4}{|c|}{ ACCENTS } \\
\hline & TRES AIGU & MOINS AIGU & $\begin{array}{c}\text { MOINS GRAVE } \\
\text { SOMBRE }\end{array}$ & PLUS GRAVE \\
\hline 3 & 10,12 & 6 & 3 & \\
\hline 4 & 10,12 & 3,6 & & \\
\hline 5 & 6,12 & 10 & 2 & \\
\hline 6 & 6 & 12 & & 3,9 \\
\hline 7 & 6 & & 3,10 & 12 \\
\hline 8 & & 2 & & $6,8,12$ \\
\hline 9 & 1 & 12 & & 6,9 \\
\hline 10 & 6 & 12 & 3 & 10 \\
\hline 11 & $2,7,12$ & & 6 & \\
\hline 12 & 6,12 & & & 3,9 \\
\hline 13 & 12 & 3,6 & 9 & \\
\hline 14 & $2,9,12$ & & 6 & 4 \\
\hline 15 & 3 & 8 & 12 & 9 \\
\hline 16 & & 4 & 12 & 9 \\
\hline 17 & 2,9 & & 6 & 12 \\
\hline 18 & 1 & & 8 & 4,12 \\
\hline 19 & & 7 & & $1,5,12$ \\
\hline 20 & & & 5 & $1,7,12$ \\
\hline 21 & 3 & 6 & & 9,12 \\
\hline 22 & 6 & & & $2,8,12$ \\
\hline 23 & & 6 & 12 & 3,9 \\
\hline 24 & & 9 & $5,6,12$ & 3,4 \\
\hline 25 & 1 & 4,9 & 6,12 & \\
\hline 26 & 1,4 & 2 & $9,6,12$ & \\
\hline 27 & 6,12 & & & 3,9 \\
\hline
\end{tabular}




\begin{tabular}{|l|l|l|l|l||}
\hline & \multicolumn{5}{|c|}{ ACCENTS } \\
\hline VERS & TRES AIGU & MOINS AI GU & $\begin{array}{c}\text { MOINS GRAVE } \\
\text { SOMBRE }\end{array}$ & PLUS GRAVE \\
\hline 28 & 12 & 3 & & 6,9 \\
\hline 29 & 6 & 9 & & 4,12 \\
\hline 30 & 7 & 6,10 & & 2,12 \\
\hline 31 & 5,7 & 2,10 & & 12 \\
\hline 32 & & 3 & 6 & 10,12 \\
\hline 33 & & 4 & & $6,8,12$ \\
\hline 34 & $3,6,9$ & & 1 & 12 \\
\hline 35 & 4 & 10 & 6 & 12 \\
\hline 36 & 6,9 & & 1 & 12 \\
\hline 37 & 6 & 12 & 1 & 9 \\
\hline 38 & 7 & 12 & $2,4,6$ & \\
\hline 39 & 8,12 & & 3,6 & \\
\hline 40 & 4 & $6,9,12$ & & \\
\hline 41 & $1,6,12$ & 8 & & \\
\hline 42 & 3,12 & 8 & & 10 \\
\hline 43 & 6 & 12 & & 3 \\
\hline
\end{tabular}

5.1. En général, d'après le tableau ci-dessus, une étude détaillêe du timbre sur la base des "accents prosodiques" nous permet de constater un systeme binaire, depuis le timbre le plus aigu jusqu'au timbre le plus grave. Cependant, dans certains vers $(3,4,5,6,8,9,10,13,15,16$, $19,21,24,25,26,28,29,30,31,32,33,35,37,38,40,41,42$ et 43) nous avons affaire à un "timbre aigu pâle". En outre, dans d'autres vers $(1,2,3,5,7,10,11,13,14,15,16,17,18,20,23,24,25,26$, $32,34,35,36,37,38,39$ et 43 ) nous avons un timbre moins grave ou sombre. 
Nous devons remarquer, également, l'absence de "timbre aigu" dans certains vers $(8,16,19,20,23,24,32,33)$, au détriment de "moins aigu".

Finalement, nous pouvons observer l'absence de "timbre grave" dans les vers suivants: $3,4,5,11,13,25,26,38,39,40,41$.

En comparant l'absence du timbre aigu (v.43) et du timbre grave (v.11), nous pourrions établir le résumé quantitatif suivant:

\begin{tabular}{|c|c|c|c|}
\hline $\begin{array}{c}\text { TIMBRES } \\
\text { AIGUS }\end{array}$ & $\begin{array}{c}\text { T. AIGUS } \\
\text { PALES }\end{array}$ & T. GRAVES & $\begin{array}{c}\text { T. SOM } \\
\text { BRES }\end{array}$ \\
\hline 35 & 8 & 32 & 11 \\
\hline \multicolumn{2}{|c|}{ Total: 43 vers } & \multicolumn{2}{|c|}{ Total: 43 vers } \\
\hline
\end{tabular}

5.2. D'après ce résultat, nous pourrions conclure que les timbres aigus et graves pourraient correspondre au flux et au reflux des vagues de l'océan, alors que le timbre aigu pâle traduirait la zone moyenne de la montée de la mer. De même, le timbre sombre signifierait la descente de la marée.

Ainsi donc, ce système binaire (aigu et grave), suivi de ses composants subsidiaires (aigus pâles et sombres) nous donnerait l'impression auditive de cette sorte de bruit monotone provoqué par le flux et le reflux des flots de l'océan. Sans oublier pour autant l'aspect visuel chromatique du blanc et du noir, en passant par le sombre ou le gris, propre de la mer de la Manche.

L'idée de mouvement, chère à Victor Hugo, est présente, sans aucun doute, dans les vers suivants:

26 "Lui, seul, battu des flots qui toujours se reforment,

27 Il s'en va dans l'abîme et s'en va dans la nuit.

28 Dur labeur! tout est noir, tout est froid; rien ne luit.

29 Dans les brisants, parmi les lames en démence,

30 L'endroit bon à la pêche, et, sur la mer immense,

31 Le lieu mobile, obscur, capricieux, changeant..." 


\section{LES IMAGES}

6.1. La présentation grammaticale de l'image.

Dans la première partie: "Il est nuit... Une femme à genoux prie, et songe, et pâlit" (v.1-12):

- dans les noms: la cabane, le logis, quelque chose (bougie), filets, pêcheur, mur, vaisselle, bahut, lit, rideaux, matelas, banc, enfants, nid, cheminée, flamme, plafond, front, femme.

- dans les adjectifs: pauvre, obscur, humble, vieux, petits, haute, sombre.

- dans des qualifications: vieux bancs, plafond sombre.

- dans un verbe: rayonne.

Le verbe être, surtout, est recherché pour l'identification métaphorique qu'il opere:

"Il est nuit. La cabane est pauvre, mais bien close.

Le logis est plein d'ombre...

Des filets de pêcheur sont accrochés au mur" (v.1-2,4)

A noter dans cette première partie, l'absence des outils de comparaison: comme, tel que, etc. On a donc affaire à une présentation abrupte de la description, mellée de sentiments visionnaires de la part de Hugo. Nous avons essentiellement deux thèmes: le monde intérieur du pêcheur et le monde extérieur, cosmique, où le phénomène météorologique annonce une certaine dramatisation du crépuscule du pêcheur, renforcé par le sème chromatique "obscur".

Grâce à un entrelacement, V. Hugo élargit progressivement le champ de sa vision: il part d'un point concret et minuscule pour aboutir à un point immense ("le sinistre océan"), appuyé de tous les éléments cosmiques: "Au ciel, aux vents, aux rocs, à la nuit, à la brume"... L'imagination de Hugo est non seulement auditive et visuelle, mais aussi et surtout dramatique, avec toute sorte de détails concernant le métier précaire du pêcheur.

C'est ainsi qu'il défend cette classe sociale si miserrable, dont le malheur touche le lecteur. C'est ainsi que l'auteur du poème nous présente une poésie sociale dont la structure profonde se trouverait dans la représentation des êtres et des choses en mouvement. Cette représentation aurait comme support l'aspect visuel et auditif. L'aspect 
chromatique teinté d'obscurité et d'ombre prend ainsi une valeur symbolique: le drame du pêcheur.

\subsection{La source des images.}

Hugo compare souvent le ciel à un plafond, à une volte. Lorsqu'il songe que l'esprit humain est captif, ce plafond est celui d'une "cabane", d'un "logis" ou d'un "cachot". (Voir, dans La légende des siècles, les poèmes de "La Fin de Satan").

De même, Lamartine:

"Et qu'est-ce que la terre? Une prison flottante,

Une demeure étroite, un navire, une tente..." (Harmonies poétiques, III,9)

Dans Victor Hugo, il y a aussi un souvenir probable de l'Arche de Noé. Les "flocons de la neige éternelle" proviennent de Lamartine encore, ou de la traduction du Livre de Job par Genoude. Les images sont empruntées à la nature.

Celles qui concernent l'être humain sont les plus nombreuses. En effet, Hugo traduit un état d'âme par un mouvement ou un état physique:

"Une femme à genoux prie, et songe, et pâlit" (v.12)

6.3. Le choix des images révèle diverses préoccupations de Victor Hugo.

D'une part, l'influence de l'environnement physique: la mer qui l'entoure; sa maison de Marine-Terrace. Mais aussi la présence de thèmes litteraires chers au poète: le bagne, les forçats (Les Misérables); les pêcheurs (Les Travailleurs de la Mer).

D'autre part, l'influence du tempérament du poète: il aime évoquer une nature tourmentée et sombre: "Le sinistre océan...". Son imagination est romantique dans le choix des thèmes d'inspiration, mais aussi dans sa manière de les traiter.

\section{LA LUMIERE}

Dans la description de la "cabane", Hugo insiste, à maintes reprises, sur l'absence de lumière. Cette absence est atténuée par une 
sorte de parallélisme que le poète trace entre le monde intérieur (l'habitat) et le monde extérieur ("ce crépuscule obscur"). Cette idée chromatique de "rouge/jaune", atténuée, correspondrait à la visualisation par Hugo de la "chandelle" ("quelque chose"):

"Le logis est plein d'ombre, et l'on sent quelque chose $/$

Qui rayonne..." (La bougie donne aussi une lumière rouge /jaune).

Tout cela dans un grand ensemble dépourvu de lumière ("Il est nuit"). On dirait donc que l'auteur essaie de faire une sorte de symbiose du micro-cosme et du macro-cosme. L'insistance, non seulement de la problématique de la présence ou de l'absence de lumière, pourrait correspondre avec la valeur symbolique de la "chaleur humaine":

"La haute cheminée où quelques flammes veillent

Rougit le plafond sombre..." (v.10)

Le cadre, décrit d'une manière extraordinaire, sur les bases visuelles et auditives, permet à Victor Hugo de nous montrer, à son tour, les vrais protagonistes de l'intérieur de la cabane:

"Et cinq petits enfants, nid d'âmes, y sommeillent" (v.9)

"Une femme à genoux prie, et songe, et pâlit" (v.12)

La place des enfants, comparés avec les petits oiseaux ("nid d'âmes") annonce l'idée Judéo-chrétienne de la Providence.

\begin{tabular}{||l|l|l||}
\hline \multicolumn{3}{|c|}{ CAUSES ET EFFETS DE LA LUMIERE } \\
\hline PRESENCE DE LUMIERE & ABSENCE DE LUMIERE & OBSCURITE \\
\hline quelque chose rayonne & plein d'ombre & il est nuit \\
\hline on distingue... & sombre & crépuscule obscur \\
\hline étincelle & à la bruma & à la nuit \\
\hline flammes & l'ondée & noir sanglot \\
\hline rougit le plafond & décembre & dans la nuit \\
\hline
\end{tabular}




\begin{tabular}{||l|l|l||}
\hline blane d'écume & hiver & tout est noir \\
\hline l'âtre & & rien ne luit \\
\hline & & obscur \\
\hline & & nuit (répété) \\
\hline
\end{tabular}

\section{CONCLUSION}

Nous avons assisté, au cours de ce poème, à l'élaboration d'une savante alchimie verbale, non point, comme certains critiques le croient, pour rendre obscur le langage (du point de vue de la suggestion, il n'y a ni clarté ni obscurité), mais pour éviter tout ce qui est explication et exploiter systématiquement les puissances de suggestion. Plutôt que de le décrire, Victor Hugo nommera l'objet; plutôt que de l'identifier, il l'entourera d'allusions culturelles.

Ainsi supprime-t-il la syntaxe sous son aspect logique, en disloquant les alliances usuelles des mots, pour leur donner une valeur stylistique personnelle. Hugo préfere une alchimie verbale, celle que Mallarmé définira comme "le langage humain ramené à son rythme essentiel"17.

Nous partageons volontiers le jugement de Banville ${ }^{18}$ quand il dit: "La Poésie est à la fois Musique, Peinture, Eloquence; elle doit charmer l'oreille, imiter les couleurs, rendre des objets visibles et exciter tous les mouvements qu'il lui plaît d'y produire; aussi est-ce le seul art, complet, nécessaire, et qui contienne tous les autres".

Victor Hugo lui-même nous fournira les mots de la fin:

${ }^{17}$ Dans sa lettre du 27 juin 1884 à M. Léo D'orfer. Citée dans le livre d'Henri Mondor: A propos de la poésie de Mallarmé, p.118.

${ }^{18}$ Dans son Petit traité de Poésie française. 
"La Poésie s'adresse d la sensibilité, non au savoir; a la connaissance intuitive, non à la raison discursive; a l'imagination, non d la logique. Elle s'efforce, non de provoquer, mais d'émouvoir et d'éveiller dans le coeur des échos prolongés".

\section{BIBLIOGRAPHIE UTILISÉE}

BONNARD, Henri (1953): Notions de style, de versification et d'histoire de la langue française. Paris: SUDEL.

DAUZAT, Albert (1947): Grammaire Raisonnée de la Langue française. Paris-Lyon: IAC.

DONOHUE-GAUDET, M.L. (1969): Le vocalisme et le consonantisme français. Paris: Delagrave.

DREYFUS François, (1968): "Le temps des révolutions (17871870)". In: Histoire Universelle. Paris: Larousse de Poche.

DUBOIS, Jean: Grammaire structurale du français:

(1965): Le nom et le pronom

(1967): Le verbe

(1969): La phrase et les transformations.

Paris: Larousse.

FRANCOIS, Frédéric (1980): Linguistique. Paris:PUF Fondamental GIRY-SCHNEIDER:"Sélection et sémantique. Classes d'objets, compléments appropriés, compléments analysables". In: Langages numéro 115, septembre 1994.

JAKOBSON, R. (1963): Essais de linguistique générale. Paris: Minuit.

LANSON, Gustave (1951): Histoire de la littérature française. Paris: Hachette.

LOGIE, P. et MOUCHEZ Ph. (1970): La technique du résumé. Paris: Cujas.

MONIER, Henri (1989): Dictionnaire de Poétique et Rhétorique. Paris: PUF. 